\title{
Le dire impossible et le devoir de dire : des gloses pour dire l'indicible
}

\author{
Lefort, Pascaline \\ Université de Picardie Jules Verne - CURAPP UMR 7319 \\ pascalinelefort@sfr.fr
}

Résumé

Si la question de la possibilité ou l'impossibilité de dire l'expérience concentrationnaire interroge l'éthique, elle pose, pour ce qui intéresse notre point de vue de linguiste, un problème aigu. Tiraillé entre le « devoir dire » pour reprendre l'expression de Gardin et le «pouvoir dire », l'ancien déporté se heurte à l'hétérogénéité du sens, à ces mots qui ne vont pas de soi, pour reprendre Authier-Revuz, à ces mots qui sont déjà habités. Aussi, comment le langage peut-il dire et dévoiler une expérience aussi traumatisante comme l'expérience concentrationnaire ? Nous verrons dans cet article dans quelle mesure la glose - ou nomination multiple - est un des moyens pour l'ancien déporté d'appréhender et de dire le vécu concentrationnaire.

Mots clés : nomination multiple, glose, témoignage, camp de concentration.

\section{Introduction}

«On peut toujours tout dire. L'ineffable dont on nous rebattra les oreilles n'est qu'alibi. Ou signe de paresse. On peut toujours tout dire, le langage contient tout » écrit Semprun dans L'écriture ou la vie (1994 : 23). Il est vrai que la parole «contient tout », qu'elle est adressée à et naît de l'appel de l'autre. Elle est aussi ce lieu du dévoilement de soi, voire de cet indicible, aujourd'hui notion complexe qu'Adorno associe à la Shoah. L'indicible, qui se définit comme ce qui ne peut être traduit par des mots, est une notion complexe qui pose un problème majeur pour nous linguistes. Il nous semble par conséquent pertinent de revenir ici sur cette notion afin d'en préciser le sens, d'analyser le champ lexical dans lequel s'inscrit l' " indicible » et de le définir par rapport à des termes qui semblent synonymes.

Emprunté au latin tardif indicibilis qui signifie « inexprimable », «l'indicible » appartenait à l'origine au vocabulaire religieux, comme le note F. Rétif dans sa préface (2004: 7). En effet, elle explique que «dans la tradition hébraïque, le nom de Dieu, le tétragramme YHWH, est imprononçable » et qu'il est donc à la fois «révélé et indicible ». Par conséquent, «Révélation et occultation, présence et absence, dicible et indicible sont indissociablement mêlés » (2004 : 7). Dans la tradition hébraïque, l' «indicible » 
était aussi très proche de la notion d' «irreprésentable » puisqu'à "l'imprononçable du nom de Dieu s'ajoutait l'interdit biblique de la représentation » (2004 : 8). F. Rétif explique également qu'au siècle des Lumières, la sécularisation de la pensée religieuse a eu de fortes répercussions sur le sens du mot « indicible ». En effet, ce dernier se vide peu à peu de son contenu originel et «renvoie moins à l'extase religieuse qu'à une intensité des sentiments, d'émotions, d'états d'âme, qui procurent à l'homme le sentiment d'ineffable... » (2004 : 7). Cette notion est aujourd'hui souvent «associée à la psychanalyse, au retour du refoulé ou à l'univers concentrationnaire, à l'horreur de la Shoah », comme l'explique J. Richard-Zappella (2007: 377). En effet, après la Seconde Guerre mondiale, 1' «indicible » fait essentiellement référence, dans l'espace franco-germanique, aux camps de concentration et à la Shoah : «le mot indicible peut difficilement être prononcé aujourd'hui sans faire référence à l'univers concentrationnaire qui a blessé le cour de l'Europe civilisée en plein XXe siècle », écrit F. Collin dans son article « L'indicible est dans le dit» (2004:61).

La notion d' " indicible » est donc une notion qui a évolué avec le temps et qui est employée dans un contexte bien particulier. En effet, si l'on s'en réfère aux dictionnaires, on constate que pour le TLF, l' « indicible» est «ce qui n'est pas traduisible en mots» et «ce qu'on ne peut pas dire » selon Le Robert (2003). Dans La lettre à Lamartine, Musset n'écrit-il pas «comment exprimerai-je une peine indicible? ». Ce terme est donc synonyme d' «indescriptible », d' «inexprimable » et suivant les lexicographes, ce terme désigne selon ses contextes et ses emplois l'impossibilité de dire une expérience limite, "l'indicible se réfère à un modèle langagier qui bloque la translation véridique d'une chose ou d'un fait donné » (M. Rinn, 1998 : 7). Cette « chose », ce « fait» est ici l'expérience concentrationnaire, expérience traumatisante et douloureuse qui ajoute à la difficulté de dire. M. Rinn remarque également, dans de nombreux articles, que selon la plupart des dictionnaires consultés, ce terme d' « indicible » est lié à des états affectifs, à la fois positifs et négatifs, auxquels on ne peut donner une expression verbale. Ainsi, Le Robert juxtapose sauvagement, comme beaucoup d'autres dictionnaires, la douleur, l'épouvante, l'angoisse, la peine, la beauté, le bonheur. M. Rinn, qui a mené une étude lexicale sur l' « indicible » à l'aide de différents dictionnaires tels que Le Robert électronique ou Le Littré, a défini ce terme par rapport à ses substituts synonymiques et a notamment déterminé les relations qu'il entretient avec des adjectifs tels qu' «ineffable », « inexprimable », «incompréhensible ». Il explique que tout comme l'adjectif « indicible », l' « ineffable » « dénomme l'incapacité de s'exprimer en paroles » (1995: 745), mais qu'à la différence de l' "indicible », elle est «lié(e) exclusivement "aux choses agréables » comme la douceur, le bonheur, les délices, l'extase, etc. »(1998 : 41). De même, M. Rinn explique à propos de l' "ineffable », que «c'est à cause de la vénération portée à un objet particulier que la parole ne doit pas être proférée. L'ineffable serait donc un interdit de verbalisation que le locuteur s'imposerait à lui-même par un acte volontariste ou qui serait imposé par une civitas donnée » (1995: 747). Par 
conséquent, contrairement à l' « indicible », la notion d' « ineffable » n'est pas appropriée pour parler de l'expérience concentrationnaire, du génocide.

Quant à l'adjectif « inexprimable », il renvoie tout d'abord d'après Le Robert à ce qui est «impossible ou très difficile d'exprimer ; qui est au-delà de toute expression » puis est lié dans la seconde acception à des états psychiques violents comme la haine, l'épouvante. Toutefois, «inexprimable» s'oppose à « indicible » dans la mesure où ce dernier «concerne uniquement l'expression verbale (1995: 747) alors qu'avec l'adjectif « inexprimable », «tous les moyens d'expressions sont défaillants à cause d'un objet insaisissable » explique M. Rinn (1995 : 747). Il en conclut d'après ses recherches qu' «il existe une différence entre l'inexprimable et l'indicible d'une part et l'ineffable d'autre part à cause de la perception de l'objet. Pour les deux premiers, celui-ci se refuse à la saisie humaine du fait de son caractère étranger. Par contre, un objet est ineffable précisément à cause de la relation privilégiée qu'il entretient avec le sujet» (1995: 747). L’adjectif « incompréhensible » signifie quant à lui «qui ne peut être compris ; dont la pensée ne peut saisir l'essence » (Le Robert) et renvoie tout d'abord à des notions abstraites telles que Dieu par exemple que l'homme ne peut appréhender. Ce n'est que dans la seconde acception que cet adjectif est lié à ce qui est «impossible ou difficile à comprendre, à concevoir, à expliquer » (Le Robert). C'est donc dans ce sens que l'on pourrait utiliser cet adjectif dans le contexte concentrationnaire. On pourrait ajouter à cette analyse le terme d' «innommable ». Cet adjectif signifie dans la première acception ce «qui ne peut être nommé» (Le Robert) et renvoie dans la deuxième acception à ce qui ne peut être désigné, nommé de part son caractère vil et ignoble. C'est dans ce deuxième sens que l'on peut comprendre l'emploi de l'adjectif « innommable ».

« Dire l'indicible » semble donc reposer sur un paradoxe. Toutefois, l'existence des témoignages montre que les rescapés des camps ont réussi à «articuler leur cri» (J. Semprun, 1994: 210), qu'ils sont parvenus à composer avec cet indicible, à en reculer les limites. Seulement, comment l'écriture permetelle de passer au-delà de l'indicible ? Quels sont les différents procédés de mise en langage de cette terrible et douloureuse expérience ?

Des recherches sur les différents modes de transmission de ce vécu ont montré que la nomination multiple était un procédé discursif récurrent au sein des témoignages étudiés. Ce foisonnement de gloses et d'autoreformulations contribue d'ailleurs, selon nous, à construire une écriture de l'indicible, une écriture de la mémoire.

Cet article, qui s'inscrit dans le cadre de l'analyse du discours et de la pragmatique, s'interrogera sur la présence et le rôle de ce métalangage. Nous verrons notamment que les nominations multiples ou la «non-cö̈ncidence » dans le dire pour reprendre Authier-Revuz (1993) reflètent la difficulté des anciens déportés à dire le vécu concentrationnaire, qu'elles sont le signe d'une non-coïncidence entre le sujet, la langue et le réel. 
Notre étude portera sur quatre récits de vie : Si c'est un homme de P. Levi, écrit juste après son retour du camp d'Auschwitz-Monowitz entre décembre 1945 et janvier 1947, l'œuvre de J. Semprun, L'écriture ou la vie, publiée en 1994. Notre étude portera également sur le récit de F. Christophe Une petite fille privilégiée, déportée enfant avec sa mère au camp de Bergen-Belsen ainsi que sur le récit de P. Seel Moi, Pierre Seel, déporté homosexuel qui a connu l'enfer des camps nazis à cause de son orientation sexuelle. A ces récits s'ajoute le roman de J. Semprun, Le Grand Voyage, écrit en 1963, dix-huit ans après la libération des camps et qui raconte le voyage en train des déportés de Paris à Buchenwald ainsi que leurs conditions de vie au sein du camp.

A travers différents exemples, nous tenterons de montrer que la nomination multiple est une des conséquences de la difficulté à dire l'expérience concentrationnaire et qu'elle permet à l'ancien déporté d'appréhender le réel, son vécu de la manière la plus juste possible ou du moins qui lui paraît être la plus juste possible, la nomination multiple étant un moyen d'accéder au mot «juste ». Nous avons ainsi relevé différentes séquences où cette nomination multiple permet de mettre en avant, à travers la diversité des effets discursifs, cette difficulté à dire, cette crainte de ne pas pouvoir trouver le mot juste.

\section{La glose-Quel type de processus discursif ?}

La glose a évolué avec le temps du point de vue de sa définition. En effet, d'un point de vue étymologique, le mot glose, du latin tardif glosa, glissa qui signifie «mot rare» et du grec glissa qui signifie «langue » et «mot rare ou archä̈que », désigne tout d'abord un mot rare qui a besoin d'être expliqué. Au fil du temps, la définition évolue et ce mot va prendre un autre sens puisqu'il ne va plus faire référence au mot difficile ou rare à expliquer mais à l'explication elle-même de ce mot. Ainsi au XVIe siècle, le mot glossaire, emprunté au latin glossarium va signifier «dictionnaire où l'on explique les mots rares ou vieillis » (Baumgartner \& Ménard, 1996 : 362). De nos jours, la glose a toujours ce sens et les dictionnaires comme Le Robert par exemple la définissent comme étant « une annotation entre les lignes ou en marge d'un texte, d'un manuscrit, pour expliquer ou interpréter un mot difficile, éclaircir un passage obscur » (2003). Elle constitue donc par extension un commentaire, une note explicative, une sorte de métalangage.

Les linguistes optent aussi pour cette définition de la glose mais la revisitent en l'appréhendant à l'aune de trois critères : sémantique (« sémantique, séquence métalinguistique portant sur un mot »), syntaxique (" en situation parenthétique ») et pragmatique («à visée explicative ») (F. Neveu, $2003: 148)$.

La glose viserait à instruire, à expliquer, à élucider des phénomènes, des vécus qui émergent difficilement. Elle permettrait de fait d'éclairer le destinataire voire à rendre intelligible ces éléments au destinateur qui décline ainsi son dire. 
Gloser, n'est-ce pas, selon les différentes définitions, faire un commentaire métalinguistique sur un mot, reformuler, dire autrement et de différentes façons une chose, un réel, le rendre intelligible ? Les gloses sont donc la trace d'une nomination multiple c'est-à-dire « une configuration énonciative consistant en une équivalence entre plusieurs manières de nommer une même chose » (S. Bikialo, 2003 : 7).

Nommer c'est se livrer à un acte énonciatif «consistant dans l'association d'un signe et d'un référent. Elle met donc en contact deux ordres distincts, l'ordre de la langue et celui des choses, du réel » (S. Bikialo, 2003 : 236). J. Authier-Revuz rappelle à ce propos que «l'énonciation - et en elle la dénomination - apparaît comme la mise en contact de deux ordres distincts, hétérogènes, celui du monde réel, et celui, non moins réel, de la langue comme ordre propre. » Rendus muets par l'horreur du vécu, J. Semprun, R. Antelme et les autres se heurtaient à l'écart entre l'expérience concentrationnaire et l'existant verbal. C'est l'énonciation, le truchement du verbal qui va permettre de nommer, de dénommer.

La «grille» de la langue, - tout à la fois extérieure au sujet et indépendante des choses s'impose au cœur de l'acte énonciatif de nomination, comme ce qui inscrit un écart au cœur même de ce qu'elle rend possible, condition de saisie du réel qui y fait en même temps obstacle (1995: 518).

J. Authier-Revuz insiste sur le fait que la langue a son ordre propre et dégagé du réel. L'intérêt de notre travail est de nous interroger sur ce qui permet l'articulation entre ces «deux ordres distincts, hétérogènes » que sont le monde réel et celui de la langue. La méconnaissance du réel de la langue, de son ordre propre et distinct du réel, a parfois conduit à envisager non pas un écart entre la langue et le réel mais au contraire une « adéquation ». P. Siblot souligne très bien cette tentation :

Nommer, c'est classer dans une catégorie linguistique en même temps qu'assigner une place dans l'ordre du monde. Aussi est-il normal qu'on ait très tôt considéré qu'un lien essentiel unit le nom à l'objet nommé, dans une relation pleinement motivée laissant peu de place à l'arbitraire. [...] Dans cette parfaite adéquation entre la chose et son nom, celui-ci se confond avec cellelà (1997 : 42).

Le rapport entre les mots et le réel c'est-à-dire ici l'expérience concentrationnaire est loin d'être «une parfaite adéquation ». En effet, dire le réel à l'aide du langage n'est jamais sans difficultés, «la langue étant une convention collective, ses unités ne peuvent rendre compte seules de l'investissement subjectif, de la multiplicité du réel. Elle manque donc toujours le réel» (S. Bikialo, 2003 : 445). 


\section{Les gloses ou comment dire l'expérience des camps}

La non-coïncidence dans le dire, sous ses manifestations multiples, est mise en œuvre dans l'écriture de cette douleur indicible. Elle s'affiche clairement dans les textes du corpus retenu et nous tenterons de voir comment elle se manifeste, quelles formes elle prend.

\subsection{Le connecteur de reformulation c'est-à-dire}

Différentes séquences ont montré que la difficulté à dire ce vécu concentrationnaire et la recherche constante du mot juste s'expriment à travers différents types de gloses et notamment par le biais du connecteur de reformulation c'est-à-dire, un indice de glose comme le note Grimaldi : «On considérera qu'il y a glose lorsque le locuteur introduit sur un segment du discours un commentaire qu'il pose comme équivalent à ce segment» (2003 : 106). Cette structure binaire tend à créer une certaine équivalence entre les termes glosés et la glose en elle-même reliés par le connecteur : "L'explication avec c'est-à-dire tend à être posée dans une relation d'équivalence, avec une glose de type didactique à visée définitionnelle » (Périchon 2003 : 98). Ce connecteur de reformulation construit en effet l'articulation entre le ou les termes glosés et la glose en elle-même c'est-à-dire le commentaire.

Ce connecteur a été relevé dans les réflexions de certains auteurs et plus particulièrement dans celles que mène Semprun dans Le Grand Voyage :

Tout à l'heure, il va ouvrir la bouche, dans un élan désespéré, «ne me laisse pas, vieux », et il va mourir, c'est-à-dire, sa mort va arriver à son terme (Le Grand Voyage 1963 : 240-241 ).

Dans cet exemple, où le narrateur revient sur la mort d'un de ses compagnons de détention, on glisse de « il va mourir» à «sa mort va arriver à son terme». A travers la glose introduite par c'est-à-dire, Semprun précise son dire. En effet, il montre que dès sa déportation, l'homme entre dans un processus de mort : perte de la dignité, déshumanisation, anéantissement physique et mort psychique. La mort c'est-àdire la cessation définitive de la vie n'apparait alors que comme le terme, le point final de ce long processus. Rappelons ici un des vers du poème de Vallejo, cité par Semprun dans L'écriture ou la vie au moment de la mort de son camarade Morales "Pero el cadáver;ay;siguió muriendo... » qui signifie « Mais le cadavre, hélas! continua de mourir» (1994: 375).

Ce type de glose apparaît également dans d'autres écrits de J. Semprun, plus particulièrement dans L'écriture ou la vie. Le connecteur de reformulation c'est-à-dire est notamment convoqué dans le récit afin de revisiter la phrase de Wittgenstein «Der Tod ist kein Ereignis des Lebens. Den Tod erlebt man 
nicht » qui signifie «La mort n'est pas un événement de la vie. La mort n'est pas une expérience vécue »:

En fait, pour être rigoureux, l'énoncé de Wittgenstein devrait s'écrire ainsi : «Mein Tod ist kein Ereignis meines Lebens. Meinen Tod erlebe ich nicht. » C'est-à-dire : ma mort n'est pas un événement de ma vie. Je ne vivrai pas ma mort (L'écriture ou la vie, 1994 : 225-226).

Nous pouvons appliquer à cet exemple le schéma suivant :

«Mein Tod ist kein Ereignis meines Lebens. Meinen Tod erlebe ich nicht» sont les termes « A » et «ma mort n'est pas un événement de $m a$ vie. Je ne vivrai pas ma mort», les termes «B ». Ces deux termes sont mis en relation d'équivalence par une jointure, dans le cas présent par c'est-à-dire.

Dans cet extrait, J. Semprun revient sur la phrase du philosophe du langage qu'il ne juge pas exacte et propose sa propre conception de la phrase. Il pense en effet qu'elle ne doit pas être une généralité parmi tant d'autres mais qu'elle doit au contraire s'appliquer à soi-même. Aussi, a-t-il fait le choix de remplacer les différents articles définis par des pronoms personnels de première personne du singulier. Ainsi, « Mein », « meines », « Meinen » et « ich » remplacent « Der », « des », « Den » et « man ». Autrement dit, en revisitant cette phrase, Semprun déclare que certes nous ne pouvons pas vivre notre propre mort mais que ce n'est pas pour autant que la mort ne peut pas être une expérience vécue. Mettre en regard l'expérience concentrationnaire et la sentence de Wittgenstein conduit J. Semprun à discuter cette conception philosophique de la mort. L'expérience concentrationnaire l'autorise à aller contre cette position philosophique, à en démontrer la non pertinence. Ainsi, il explique

"La mort n'est pas un événement de la vie. La mort ne peut être vécue", avait écrit ce con de Wittgenstein. J'avais vécu la mort de Morales, pourtant, j'étais en train de la vivre. Comme j'avais, un an auparavant, vécu la mort de Halbwachs. Et n'avais-je pas vécu de même la mort du jeune soldat allemand qui chantonnait La Paloma ? La mort que je lui avais donnée ? N'avais-je pas vécu l'horreur, la compassion, de toutes ces morts? De toute la mort? La fraternité aussi qu'elle mettait en jeu (1994:252).

\subsection{Les autoreformulations avec verbes de parole}

Cette volonté de dire juste s'exprime également à travers d'autres types de gloses. Si dans les exemples précédents, on se trouvait face à des gloses introduites par le connecteur de reformulation c'est-à-dire, les autoreformulations avec verbe de parole rencontrées dans le corpus sont également, selon nous, des 
indices de nomination multiple et de cette difficulté à faire coïncider le langage et le réel et à dévoiler les souvenirs de cette expérience. Dans ce type de glose, le locuteur reprend son propre discours en y ajoutant des verbes du dire sous des formes de type : «je veux dire $», ~ « j$ 'entends dire $» \ldots$

\subsubsection{Je veux dire}

Deux autoreformulations de ce type ont été relevées dans les réflexions de Semprun sur le statut des déportés :

Le surlendemain, donc, j'ai vu apparaître le nom de Halbwachs sur la liste des décès quotidiens. J'ai pris dans le fichier central de l'Arbeitsstatistik le casier correspondant à son matricule. J'ai sorti la fiche de Maurice Halbwachs, j'ai effacé son nom : un vivant pourrait désormais prendre la place de ce mort. Un vivant, je veux dire : un futur cadavre (L'écriture ou la vie 1994 : 62).

Si l'autoreformulation de type « je veux dire » montre d'une part que le terme choisi « vivant» n'est pas approprié pour désigner le déporté, elle va d'autre part, permettre à Semprun de mieux dire, de trouver des mots plus justes et plus aptes, plus proches du réel vécu. En effet, à travers cette autoreformulation, l'écrivain montre qu'à l'intérieur du camp le déporté n'est plus un être «vivant» mais un mort «en puissance » puisque le décès du déporté est quasiment inéluctable.

Un autre exemple de ce type apparaît un peu plus loin dans le récit :

Imprimé à l'avance, 44904 était le matricule qui m'était destiné. Je veux dire : qui était destiné au déporté, quel qu'il fût, qui serait arrivé à ce moment précis devant l'homme chargé de remplir cette fiche (L'écriture ou la vie 1994 : 382).

Ces différentes autoreformulations montrent que l'auteur, en couchant sur le papier son récit, prend conscience de l'inadéquation de son dire, du fossé entre sa parole et le «réel » où son moi est né. L'être humain qu'est l'auteur n'existe plus dès qu'il a franchi les portes du camp, le matricule participant à la destruction de l'identité, du «moi» du prisonnier. L'autoreformulation est une autre manière pour Semprun de dire cette destruction en termes voilés mais aussi de décrire une réalité.

\subsubsection{J'entends dire}

Ce type de glose a été relevé dans Si c'est un homme de Levi : 


\begin{abstract}
A la sortie du KB., si on ne dispose pas de protections particulières, on n'est pas réinséré dans son Block et dans son Kommando d'origine, mais, sur la base de critères que j'ignore, on est affecté à n'importe quelle autre baraque et dirigé vers n'importe quel travail. Bien plus, on sort nu du K.B. ; on reçoit des vêtements et des souliers « neufs » (j'entends dire différents de ceux qu'on y a laissés à l'entrée), qu'il faut s'employer avec zèle et rapidité à adapter à sa propre personne, ce qui implique de la fatigue et des dépenses (Si c'est un homme 1987 : 83).
\end{abstract}

L'autoreformulation de type «j'entends dire » permet à Levi de revenir sur le terme « neufs » afin de préciser ce qu'il entend réellement par cet adjectif, «il suffit d'une incompréhension, inquiétude, besoin...et le langage est rappelé » (A. Rey, 1974 : 44). En effet, le terme « neufs » ne désigne pas «ce qui vient d'être fait et qui n'a pas encore servi» (Le Robert) mais a le sens de « différents ». Les guillemets sont d'ailleurs l'indice que le terme employé « neufs » n'est pas à prendre dans son habituel. Cette autoreformulation permet donc à Levi de préciser son dire, de dépasser le sens commun qui circule hors des murs du camp.

Si tout mot est habité, comme le dit Bakhtine, tout mot qui renvoie à une situation, à un moment, à un vécu du camp prend une toute autre charge qui ne se superpose pas à la tonalité commune mais la réinvestit totalement.

\title{
2.3. Gloses et figures de correction
}

Les gloses analysées jusqu'à présent s'apparentent à des figures de répétition, le locuteur répétant, redoublant des termes afin d'en donner l'équivalent et de tenir des propos les plus justes, moins inappropriés. Ces nominations multiples seraient ainsi en lien avec une figure de rhétorique, la métabole qui est selon Fontanier

bien moins la variété des mots dans les sons qui frappent l'oreille, que dans les nuances qui frappent l'esprit, c'est cet enchérissement de chaque nouveau synonyme sur celui qui précède, et cet effet toujours croissant de l'un à l'autre jusqu'au dernier (1977 : 332-333).

Cette figure de rhétorique pourrait être mise en relation avec une autre figure, l'expolition, que Morel classe parmi les figures qui permettent de «s'assurer que la communication passe bien ou qu'il n'y a pas de malentendu » en ce qu'elle « réexpose de façon plus nette la pensée » de l'énonciateur (1982: 13).

Toutefois, en dehors de ces figures, la nomination multiple apparaît également sous des formes qui rappellent cette autre figure rhétorique, l'anthorisme. Il s'agit, selon Molinié de 
faire se succéder deux notations qui se rectifient, l'une niant l'autre, pour achever sur l'indication la plus forte ou la plus adéquate à l'effet de sens à produire. L'anthorisme le plus net, le plus massif aussi, apparaît donc dans la succession d'une expression positive et d'une expression négative, ou l'inverse (il n'est pas fou : il est fou à lier). Mais on peut le trouver également dans la succession de deux termes qui soient chacun positifs (ou négatifs), mais de telle manière que le second apparaisse comme la négation du premier. [...] L'anthorisme, toujours surprenant et efficace, sert éventuellement de support à l'épanorthose (1992: 54).

En effet, ces ajustements ou autres corrections ne peuvent pas ne pas faire penser à l'épanorthose, figure discursive qui permet à l'orateur de rétracter ou corriger " quelque chose de ce qu'il a déjà avancé, \& qui lui paroît trop foible: il y ajoûte quelque chose de plus énergique, \& de plus conforme à la passion qui l'occupe ou le transporte. Voyez CORRECTION» (L'Encyclopédie, 1750).

Le marqueur de glose "ou plutôt », qui balise certain des textes, est selon nous une des traces d'une nomination multiple, d'une correction, d'une rectification. En effet, «ou plutôt » entraîne certes une opération de reformulation mais se différencie des autres marqueurs cités dans la mesure où, comme le notent Soublin et Tamine, "ou plutôt» "au moment même où il pose l'équivalence des deux éléments supprime le premier» (1973: 242). Authier-Revuz propose, quant à elle, une autre analyse de ce marqueur de glose, il n'y a pas, selon elle, de suppression de la première nomination par la seconde, le deuxième dire est considéré comme " préférable, sans pour autant « effacer » le premier dont il est donné non comme remplaçant mais comme alternative » (1995:620).

Un exemple de ce type apparaît dans Si c'est un homme de Levi :

Tous les « musulmans » qui finissent à la chambre à gaz ont la même histoire, ou plutôt ils n'ont pas d'histoire du tout : ils ont suivi la pente jusqu'au bout, naturellement, comme le ruisseau va à la mer. Dès leur arrivée au camp, par incapacité foncière, par malchance, ou à la suite d'un incident banal, ils ont été terrassés avant même d'avoir pu s'adapter (1987 : 138).

La glose a effectivement ici une fonction de rectification. En effet, le marqueur de glose «ou plutôt » permet le passage de «ont la même histoire » à «ils n'ont pas d'histoire du tout». En optant pour une tournure négative, Levi cherche à se rapprocher de la véritable condition des «musulmans » c'est-à-dire les détenus les plus faibles et les plus en danger. En effet, dès leur arrivée au camp, leur histoire s'arrête et leur mort est malheureusement inéluctable. La glose permet de plus à Levi de poétiser la vie des 
« musulmans » en créant une analogie entre leur destin et celui d'un ruisseau. Tout comme le ruisseau qui n'a pas d'autre issue, naturellement, que de rejoindre la mer, le «musulman » est condamné a une mort certaine.

\subsection{Gloses et récapitulation}

Certaines gloses que nous avons rencontrées peuvent avoir une fonction de récapitulation. C'est notamment le cas des gloses introduites par des marqueurs de type en somme, bref et en un mot. Ces derniers sont des marqueurs de reformulation mais se situent dans un autre registre que c'est-à-dire par exemple. En effet, alors que les marqueurs de type c'est-à-dire «lient deux constituants de même niveau hiérarchique et indiquent une relation d'équivalence sémantique ou de paraphrase entre deux formulations » (M.M.G. Negroni, 1999 : 173), les marqueurs comme bref font partie des opérations dite de récapitulation dont l'usage permet au locuteur « de revenir sur sa première formulation afin d'en tirer l'essentiel» (C. Rossari, 1993 : 18). Le locuteur qui utilise ce type de marqueur vient donc «compléter après coup un constituant présenté pourtant comme se satisfaisant à lui-même dans un premier temps » (E. Roulet, 1987 : 115-116) et vise à marquer son changement de perspective par rapport au discours antérieur.

Le marqueur en somme apparaît notamment dans Une petite fille privilégiée de F. Christophe :

Nous avons de plus en plus de poux. Pas de puces comme en France, mais de plus en plus de poux de tête, des poux de corps reconnaissables à une espèce de croix gammée sur le dos. En somme, des poux boches (1996: 96).

Le marqueur de glose en somme permet ici à F. Christophe de revenir sur ses premiers dires « des poux de tête, des poux de corps reconnaissables à une espèce de croix gammée sur le dos » afin de les récapituler, de les résumer en une seule formule "poux boches». A travers le choix de cette formule, l'auteure réveille la petite fille qui est en elle, cette petite fille qui a connu l'enfer des camps et qui attribuait à des insectes des qualités humaines, en l'occurrence ici une nationalité. Cette glose est aussi un moyen pour elle de dire la peur et le dégoût.

Si le marqueur en somme permet au locuteur de synthétiser son dire en une formule plus concise, plus juste, les gloses introduites par bref jouent également ce rôle. Un exemple de ce type a été relevé dans l'œuvre de P. Seel, Moi, Pierre Seel, déporté homosexuel : 
Il faudra dire un jour la monstrueuse histoire qui fut celle des «malgré nous », ces natifs d'Alsace et de Lorraine enrôlés pour tuer des résistants, des antifascistes et leurs familles, bref, pour assassiner les ennemis du Reich (1994: 74).

Bref permet ici le passage de différentes catégories d'opposants « les résistants », les « antifascistes » à un terme plus générique, qui englobe la totalité des opposants à savoir « ennemis du Reich ». Cet adverbe permet également le passage du verbe «tuer» au verbe «assassiner». En effet, les «malgré nous » avaient la mission de tuer les opposants au régime hitlérien, il y a donc préméditation d'où l'usage du verbe « assassiner ».

\subsection{Gloses et spécification du sens}

Hormis les figures de répétition, de correction et de récapitulation que nous avons évoquées, la peur du non bien dit et la recherche constante du bien dire s'expriment également à travers des gloses de spécification du sens de type « $\mathrm{X}$ au sens de » qui marquent la non-coïncidence du mot par rapport à luimême (Authier-Revuz 1995). Ces gloses orientent les destinataires sur le sens à donner au mot. Ainsi, en utilisant ce type de glose, l'énonciateur revient sur son dire afin que ses propos soient correctement compris et oriente «la valeur sémantique qu’il souhaite donner au mot employé » (Périchon 2003 : 101102). Une glose de ce type a été relevée dans le récit de Christophe Une petite fille privilégiée au sein d'une discussion entre l'auteure et d'autres déportés :

«-Et Jeannine G...?

- Morte à l'infirmerie. Comme cobaye humain (Je me souviens bien, elle avait vingt ans en quittant la France).

Et Guy? dis-je timidement.

En fumée. Au sens propre » (1996:130).

La forme « au sens propre » relève de la glose sémantique. Elle retient une acception sans pour autant évaluer cette acception. On assiste ici à un besoin de réduire au maximum la polysémie de la locution « en fumée », de la désambiguïser. Par conséquent, la «sélection d'un sens "propre » se fait par l'élimination implicite des autres sens possibles (vs sens figuré) »(Périchon 2003 : 103). L’ajout de la glose «au sens propre», qui renforce ici l'horreur contenue dans les termes «en fumée », replonge l'auteure dans la violence de l'expérience et de cette mort par crémation. 


\section{Conclusion}

Dire cet indicible est donc de l'ordre du possible. Cette mise en mots et en discours de l'indicible passe par la nomination multiple, la monstration de l'hétérogénéité entre la langue, entre les mots et les choses. Le dire du témoignage sur l'expérience concentrationnaire semble donc reposer sur ce métalangage ; la douleur de ce vécu trop longtemps emprisonnée, enfouie explose en une succession de mots, d'expressions qui «[...] s'interprètent comme autant de symptômes par lesquels le sujet parlant tente de négocier l'impossible unicité de son discours » (Sarfati 1997 : 74).

La nomination multiple permet ainsi d'exhiber le déploiement de la pensée en train de se dire, à la recherche du mot qui serait juste. Elle est la marque de la non-coïncidence des mots et du vécu, des mots à eux-mêmes et dévoile à travers ces procès leur caractère manquant; elle permet de dire à travers les mots ce qui échappe aux mots.

Il ne faut donc pas voir dans la nomination multiple le signe d'une «défaillance d'un locuteur » pour reprendre Authier-Revuz (2000 : 37) ou d'une impuissance linguistique ; elle est au contraire le signe de la non-coïncidence entre le sujet, la langue et le réel.

\section{Références bibliographiques}

Auchlin, A. (1993). Au petit bonheur du Bien Dire - Note sur le traitement du « Bien Dire » en analyse pragmatique $\mathrm{du}$ discours. Le Bien dire, Cahiers de Praxématique, n²0, 45-63.

Authier-Revuz, J. (1993). Du je de l'intention au jeu du hasard: figures méta-énonciatives du «bien-dire».

Cahiers de Praxématique, $n^{\circ} 20,87-113$.

Authier-Revuz, J. (1995). Ces mots qui ne vont pas de soi, Boucles réflexives et non coüncidences du dire. Paris: Larousse.

Authier-Revuz, J. (2000). Deux mots pour une chose : trajets de non-coïncidence. Annales Littéraires, $n^{\circ} 701$, série Linguistique et Sémiotique, $n^{\circ} 39,37-61$.

Baumgartner, E. \& Ménard Ph. (1996). Dictionnaire étymologique et historique de la langue Française. Paris : Librairie Générale Française.

Bikialo, S. (2003). Plusieurs mots pour une chose - De la nomination multiple au style de Claude Simon. Thèse de Doctorat. Paris III.

Christophe, F. (1996). Une petite fille privilégiée, Une enfant dans le monde des camps 1942-1945. Paris: L'Harmattan.

Collin, F. (2004). L'indicible est dans le dit. L'indicible, 55-63.

Fontanier, P. (1977) [1821]. Les Figures du discours. Paris : Flammarion. 
Gardin, B. (1993). Le bien dire : essai de circonscription. Cahiers de Praxématique, $n^{\circ}$ 20, 27-43.

Grimaldi, E. (2003). Glose et effets de glose dans les Mémoires d'Outre-Tombe et autres textes de Chateaubriand. Le mot et sa glose, 105-127.

Lacroix, M. (1990). De la Politesse. Paris : Julliard.

Le Robert électronique. (1992). Paris : Dictionnaires Le Robert [RobE.]

Levi, P. (1987). Si c'est un homme. Paris : Editions Julliard.

Levi-Valensi, J. (1999). Ce qu'« il est possible de dire». Problématique des genres, problème du roman, Etudes réunies par Jean Bessière et Gilles Philippe, 257-267.

Littré, E. (1877). Dictionnaire de la langue française. Paris : Hachette.

Molinié, G. (1992). Dictionnaire de rhétorique. Paris : Librairie Générale Française.

Morel, M-A. (1982). Pour une typologie des figures de rhétorique : points de vue d'hier et d'aujourd'hui. DRLAV, $n^{\circ}$ $26,1-62$.

Negroni, M.M.G. (1999). Réinterprétations et connecteurs : le cas des connecteurs enchérissants et des connecteurs reformulatifs. Pragmatics en 1998, Selected Papers From The 6th International Pragmatics Conference, vol.2, $172-180$.

Neveu, F. (2003). La glose et le système appositif. Le mot et sa glose, 143-167.

Périchon, B. (2003). La glose, un procédé discursif à double détente : asseoir ses propres dires, dénoncer les dires de l'autre. Le mot et sa glose, 93-104.

Rétif, F. (2004). Préface. L'indicible, 7-8.

Rey, A. (1974). Sens et discours poétique chez Valéry. Paul Valéry contemporain, Actes et colloques, $n^{\circ} 12,39-48$.

Richard-Zappella, J. (2007). Lorsque de l'indicible naît le désir de dire. Actes de Colloque, Confidence/Confiding Dévoilement de soi dans l'interaction/self disclosure in interaction, 377-390.

Rinn, M. (1995). Approches de la sémantique lexicale de l'indicible. Colloque de la relève suisse sur la théorie littéraire, 741-753.

Rinn, M. (1998). Les récits du génocide. Sémiotique de l'indicible. Lausanne-Paris : Delachaux et Niestlé S.A.

Robert P. (2003). Le Nouveau Petit Robert. Paris : Le Robert.

Rossari, C. (1993). Les opérations de reformulation. Analyse du processus et des marques dans une perspective contrastive français-italien. Berne : Peter Lang.

Roulet E. (1987). Complétude interactive et connecteurs reformulatifs. Cahiers de Linguistique Française 8, 111140.

Sarfati, G-E. (1997). Eléments d'analyse du discours. Paris : Nathan Université.

Seel, P. (1994). Moi, Pierre Seel, déporté homosexuel. Paris: Editions Calmann-Lévy.

Semprun, J. (1994). L'écriture ou la vie. Paris: Editions Gallimard.

Semprun, J. (1963). Le Grand Voyage. Paris: Editions Gallimard.

Siblot, P. (1997). Nomination et production de sens : le praxème. Langages, $n^{\circ} 127,38-55$.

Soublin, F. \& Tamine J. (1973). Métaphore et cadres syntaxiques = la juxtaposition. Le Français moderne, XLI, $\mathrm{n}^{\circ} 3$, 240-255

Trésor de la langue française. (1971-1994). Paris : Editions du CNRS. 
(C) aux auteurs, publié par EDP Sciences, 2012 